\title{
A Local Region Molecular Dynamics Simulation Method for Nanoscale Sliding Contacts
}

\author{
Rui-Ting Tong ${ }^{1 *}$, Geng Liu ${ }^{1}$ and Tian-Xiang Liu ${ }^{2}$
}

\begin{abstract}
Computational efficiency and accuracy always conflict with each other in molecular dynamics (MD) simulations. How to enhance the computational efficiency and keep accuracy at the same time is concerned by each corresponding researcher. However, most of the current studies focus on MD algorithms, and if the scale of MD model could be reduced, the algorithms would be more meaningful. A local region molecular dynamics (LRMD) simulation method which can meet these two factors concurrently in nanoscale sliding contacts is developed in this paper. Full MD simulation is used to simulate indentation process before sliding. A criterion called contribution of displacement is presented, which is used to determine the effective local region in the MD model after indentation. By using the local region, nanoscale sliding contact between a rigid cylindrical tip and an elastic substrate is investigated. Two twodimensional MD models are presented, and the friction forces from LRMD simulations agree well with that from full MD simulations, which testifies the effectiveness of the LRMD simulation method for two-dimensional cases. A threedimensional MD model for sliding contacts is developed then to show the validity of the LRMD simulation method further. Finally, a discussion is carried out by the principles of tribology. In the discussion, two two-dimensional full MD models are used to simulate the nanoscale sliding contact problems. The results indicate that original smaller model will induce higher equivalent scratching depth, and then results in higher friction forces, which will help to explain the mechanism how the LRMD simulation method works. This method can be used to reduce the scale of MD model in large scale simulations, and it will enhance the computational efficiency without losing accuracy during the simulation of nanoscale sliding contacts.
\end{abstract}

Keywords: Local region molecular dynamics simulation, Nanoscale, Sliding contacts, Contribution of displacement

\section{Introduction}

Computational efficiency and accuracy are two main problems in many numerical simulation processes, and they always conflict with each other. Especially, how to enhance the computational efficiency is a key problem in molecular dynamics (MD) simulation, while the accuracy should be acceptable at the same time.

Many MD algorithms and hybrid methods were developed in the past years to reduce CPU cost in MD simulations. For MD algorithms, some of them focused on

\footnotetext{
*Correspondence: tongruiting@nwpu.edu.cn

${ }^{1}$ Shaanxi Engineering Laboratory for Transmissions and Controls, Northwestern Polytechnical University, Xi'an 710072, China Full list of author information is available at the end of the article
}

improving performance on one computer, including Verlet table [1], Cell-Linked list [2], Verlet cell-linked list [3] and Tabulated potential method [4], etc. Verlet table [1] calculated the distance between one atom and atoms in its radius of neighborhood, so it could reduce CPU time and improve computational efficiency. Compared with the Verlet table, Cell-Linked list [2] meshed the system according to the positions of all atoms, and it did not need to form a table or update the table during the simulation process, which showed its advantage when the model was more than 10 thousand atoms. Combined Verlet table and Cell-Linked list, the meshes were used to update the table in Verlet cell-linked list [3], and one did not need to calculate the distance of all the atoms. Therefore, for the same model, Verlet cell-linked 
list got the best computational efficiency among the three methods [5]. In MD simulations, the most time-consuming process was computing the inter-particle potential and the forces, so potentials and forces were stored in memory to save CPU time in Tabulated potential method [4]. For simple potential function, this method could not enhance computational efficiency. Using cell decomposition and data sorting method, Yao et al. [6] developed an algorithm which enhanced the neighbor list construction speed and reduced the CPU data cache miss rate. The results showed that the computational efficiency of the algorithm was 2-3 times higher than the conventional algorithms. Mason [7] presented an algorithm which did not need to construct or update the neighbor list of each atom, and the time cost was lowered by a factor of 2-3 compared with traditional algorithms. For the systems including many atoms, a modified cell-linked list method was used to reduce the number of unnecessary internuclear distance calculations by reducing the cell size in Ref. [8]. At the same time, reducing the cell size would enhance the memory requirement of computers, which might influence the computational efficiency for different systems. Nagai et al. [9] developed a mass scaling replica-exchange molecular dynamics (MSREMD) method to improve numerical stability of simulations, and the replica-exchange routine could be simplified by eliminating velocity scaling. Parallel algorithms which could enhance the simulation scale by running on multi-CPUs were also widely developed, such as force decomposition [10], static load balancing method [11], domain decomposition [12] and tree decomposition [13], etc. Both serial algorithms and parallel algorithms above improved the computational efficiency for the systems modeled by the authors without changing the simulation scale. If the scale of the MD model could be reduced, the algorithms would be more meaningful.

In order to reduce the scale of MD model without losing accuracy, multiscale methods [14-16] and multiresolution methods [17-19] which coupled MD simulation and continuum methods were developed, such as bridging scale method [20,21], bridging domain method [22, 23], coupled atomistic and discrete dislocation (CADD) method [24], multiscale boundary condition method [25], hybrid method [26] and adaptive multiscale method [27], etc. For the multiscale methods mentioned above, MD simulations were only used in part of the model where detailed behaviors of atoms should be concerned, so the multiscale methods could enhance the computational efficiency more effectively and keep computational accuracy. According to the descriptions of the references above, computational speed of the bridging scale method was two or three times faster than full MD simulation, and the multiscale boundary condition method [25] could reduce $6 / 7$ of the computational time compared with full MD simulation. Surprisingly, the time cost of the hybrid method [26] was only $1 / 20$ of full MD simulation. Furthermore, the similar methods have been used to solve nanoscale sliding contact problems [28].

Although these multiscale methods could save much CPU time, the scale of the MD region could not be changed once the model was determined. If a local MD region moves together with the sliding of the contact body, the simulation process will be more approximate to the real one, and the dimension of the model could be reduced further. Maekawa et al. [29] developed an area-restricted molecular dynamics (ARMD) simulation method to investigate the friction and tool wear in nanoscale machining. In ARMD simulation method, the restricted region moved together with the tool advancement, and the results were compared with that from full MD simulation. Pandurangan et al. [30] presented a novel multiscale model to perform nanoscratch simulations. For this novel multiscale method, an MD region with constant size translated with the indenter, and other regions were modeled by meshless Hermite-Cloud method. The results from the two methods $[29,30]$ above did not agree well with that from full MD simulation. Therefore, it will be meaningful to develop an approach which can obtain well-agreed results besides reduction of the CPU time.

In this paper, a local region molecular dynamics (LRMD) simulation method is presented based on the contribution of displacement (COD) to solve nanoscale sliding contacts between a rigid cylindrical tip and a smooth surface. A criterion is given to determine the scale of local region, and the sliding processes are carried out between the tip and the local region. Two twodimensional and one three-dimensional sliding contact models with different scale are simulated by using both the LRMD simulation and full MD simulation. For these three models, the friction forces obtained from LRMD simulation agree well with that from full MD simulation, which testifies the effectiveness of the LRMD simulation method.

\section{Model Descriptions}

For a two-dimensional nanoscale sliding contact problem, an MD model is presented in Figure 1. The rigid cylindrical tip consists of 4 layers with 120 atoms, and its radius is $R=30 r_{0}$ ( $r_{0}$ is the Lennard-Jones parameter, $r_{0}=0.2277 \mathrm{~nm}$ [31] here). Two different scales of the substrate are used in this paper: Model I: 9153 atoms (81 layers in $y$ direction and 113 atoms per layer in $x$ direction); Model II: 37001 atoms (163 layers and 227 atoms per layer). Initial gap between the tip and substrate is $d_{\mathrm{g}}=2.5 r_{0}$. 


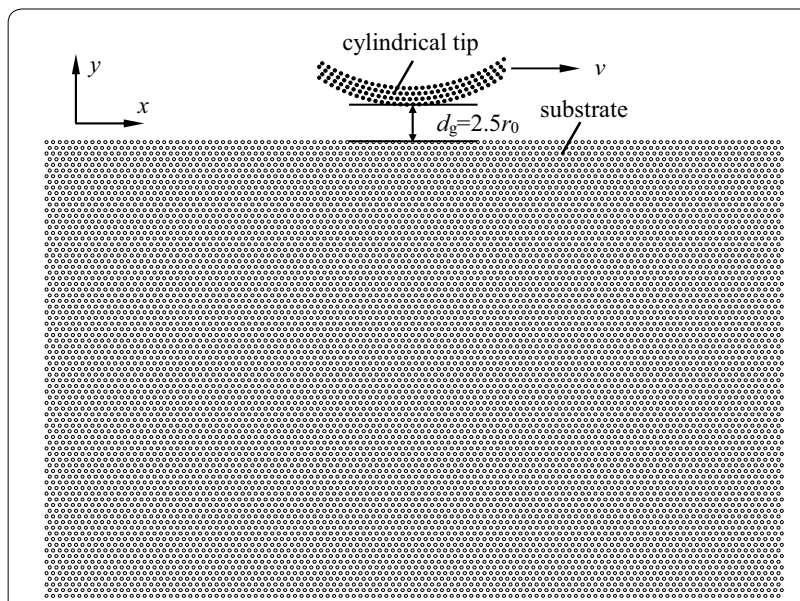

Figure 1 Two-dimensional nanoscale sliding contact model between a rigid cylindrical tip and an elastic substrate

For two-dimensional MD simulations in this paper, a classical Lennard-Jones (L-J) potential is used to describe the interactions between all atoms:

$$
U\left(r_{i j}\right)=4 \varepsilon\left[\left(\frac{r_{0}}{r_{i j}}\right)^{12}-\left(\frac{r_{0}}{r_{i j}}\right)^{6}\right], \quad r_{i j} \leq r_{\mathrm{c}}
$$

where $r_{i j}=\left\|\boldsymbol{r}_{i}-\boldsymbol{r}_{j}\right\|$, is the distance between atoms $i$ and $j, \varepsilon$ and $r_{0}$ are the L-J parameters. For FCC Cu, $\varepsilon=6.648 \times 10^{-20} \mathrm{~J}, r_{0}=0.2277 \mathrm{~nm}$ [31]. Table 1 lists the relationships between the reduced units and the Systeme International (SI) units used in this paper. The cut-off radius, $r_{c}$, is taken to be $2.2 r_{0}$ here. Fixed boundary conditions are applied along the bottom layer, and periodic boundary conditions are applied along the left and right boundaries of the substrate. The force that atom $j$ exerts on atom $i$ can be written as follows:

$$
\begin{aligned}
\boldsymbol{F}\left(r_{i j}\right) & =-\nabla U\left(r_{i j}\right) \\
& =\left(\frac{48 \varepsilon}{r_{0}^{2}}\right)\left[\left(\frac{r_{0}}{r_{i j}}\right)^{14}-\frac{1}{2}\left(\frac{r_{0}}{r_{i j}}\right)^{8}\right] \boldsymbol{r}_{i j}, \quad r_{i j} \leq r_{\mathrm{c}}
\end{aligned}
$$

Velocity-Verlet algorithm [32] is used to calculate the coordinates, the velocities, and the accelerations of all atoms:

$$
\begin{aligned}
\boldsymbol{r}(t+\Delta t) & =\boldsymbol{r}(t)+\boldsymbol{v}(t) \Delta t+\frac{1}{2} \boldsymbol{a}(t) \Delta t^{2} \\
\boldsymbol{v}(t+\Delta t / 2) & =\boldsymbol{v}(t)+\frac{1}{2} \boldsymbol{a}(t) \Delta t \\
\boldsymbol{a}(t+\Delta t) & =-\frac{1}{m} \nabla U(\boldsymbol{r}(t+\Delta t)) \\
\boldsymbol{v}(t+\Delta t) & =\boldsymbol{v}(t+\Delta t / 2)+\frac{1}{2} \boldsymbol{a}(t+\Delta t) \Delta t
\end{aligned}
$$

where $\Delta t$ is the time step, $\boldsymbol{r}$ is the coordinate vector, $\boldsymbol{v}$ is the velocity vector, and $\boldsymbol{a}$ is the acceleration vector, $m$ is the mass of an atom.

The local region molecular dynamics (LRMD) simulation method is achieved through the contribution of displacement (COD) of atoms on the centerline of the substrate. The COD $C_{\mathrm{d}}$ is defined as follows:

$$
C_{\mathrm{d}}=\frac{\sum_{i=1}^{k} u_{i}}{\sum_{i=1}^{n} u_{i}}
$$

where $u_{i}$ is the displacement component in the indentation direction of the center atom on the $i$ th layer (numbered from the topmost layer to the bottom layer), $k$ is the number of included layers in local region, and $n$ is the total number of layers included in the substrate.

The flowchart for the LRMD simulation method is given in Figure 2. Indentation process is carried out first before sliding contact, and full MD simulation is used. When the indentation is finished, a local region is chosen according to $C_{\mathrm{d}}$. Calculating the $C_{\mathrm{d}}$ until $C_{\mathrm{d}} \geq 95 \%$ corresponding to $i=k$, then $k$ layers atoms in the indentation direction will be chosen to form a local region model which will be used to simulate the sliding contact processes.

Table 1 The reduced units and SI units of the L-J potential and EAM potential

\begin{tabular}{lllll}
\hline Parameters & \multicolumn{2}{l}{ L-J potential } & & \multicolumn{2}{l}{ EAM potential } \\
\cline { 2 - 3 } & Reduced units & SI units & Reduced units & SI units \\
\hline Mass $(\mathrm{kg})$ & $\mathrm{m}$ & $1.055 \times 10^{-25}$ & $\mathrm{~m}$ & $1.055 \times 10^{-25}$ \\
Length $(\mathrm{m})$ & $r_{0}$ & $2.277 \times 10^{-10}$ & $\mathrm{~d}$ & $2.556 \times 10^{-10}$ \\
Energy $(\mathrm{J})$ & $\varepsilon$ & $6.648 \times 10^{-20}$ & $\mathrm{eV}$ & $1.602 \times 10^{-19}$ \\
Time $(\mathrm{s})$ & $\sqrt{\mathrm{mr_{0 } ^ { 2 } / \varepsilon}}$ & $2.869 \times 10^{-13}$ & $\sqrt{\mathrm{md} / \mathrm{eV}}$ & $2.024 \times 10^{-13}$ \\
Force $(\mathrm{N})$ & $\varepsilon / r_{0}$ & $2.920 \times 10^{-10}$ & $\mathrm{eV} / \mathrm{d}$ & $6.268 \times 10^{-10}$ \\
Temperature $(\mathrm{K})$ & $\varepsilon / \mathrm{kB}_{\mathrm{B}}$ & $4.815 \times 10^{3}$ & $\mathrm{eV} / \mathrm{kB}_{\mathrm{B}}$ & $1.160 \times 10^{4}$ \\
Stress $(\mathrm{Pa})$ & $\varepsilon \cdot r_{0}^{-3}$ & $5.631 \times 10^{9}$ & $\mathrm{eV} \cdot \mathrm{d}^{-3}$ & $9.594 \times 10^{9}$ \\
\hline
\end{tabular}




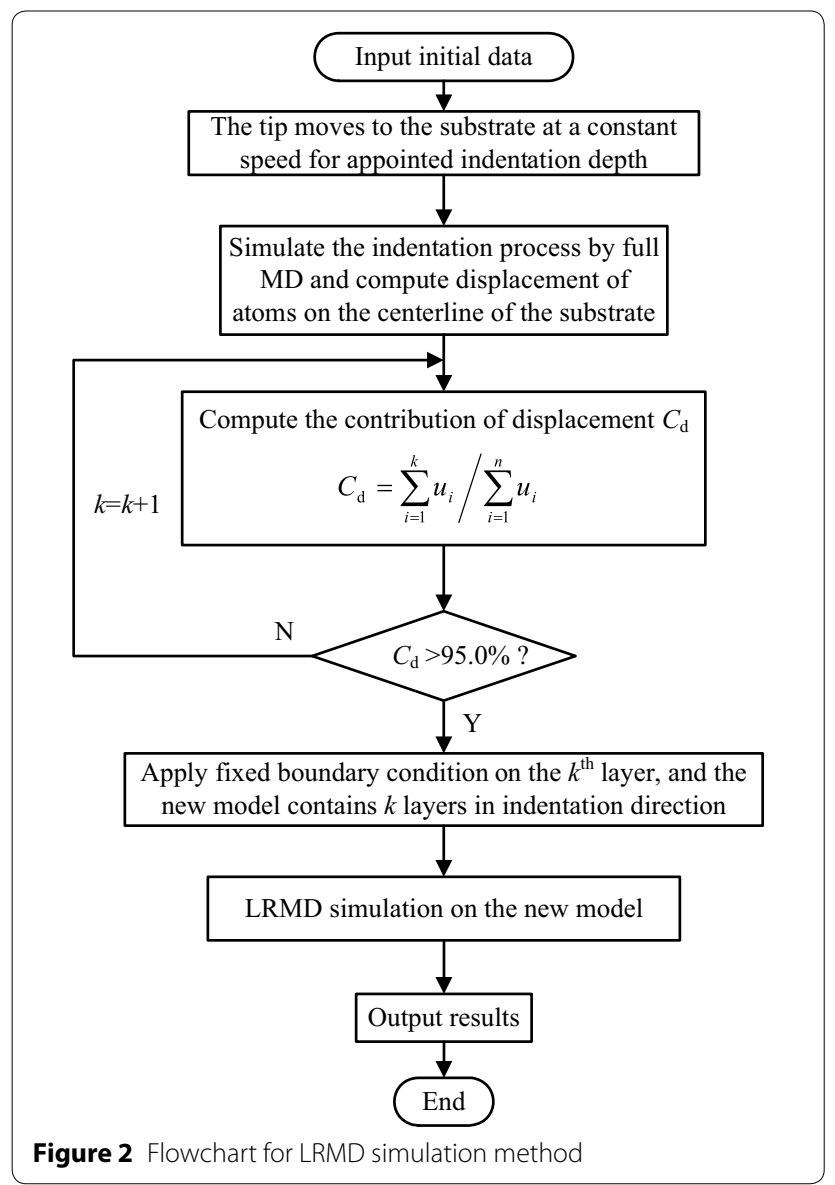

\section{Results and Discussion}

For two-dimensional MD simulations in this paper, velocity-Verlet algorithm is carried out with a fixed time step, $\Delta t=0.95 \mathrm{fs}$. Initially, velocities of all the atoms except fixed boundary ones are set with random Gaussian distribution under an equivalent temperature $T=300 \mathrm{~K}$. The system will be relaxed for $2000 \Delta t$ to reach its minimum energy configuration before loading. Then, the tip moves to the substrate with a displacement increment $\Delta s=0.025 r_{0}$ and indentation speed keeps at $v=1.0 \mathrm{~m} / \mathrm{s}$. The indentation depth is $d_{\mathrm{I}}=2.5 r_{0}$. When the indentation is finished, the tip slides along $x$ direction. A high sliding speed could induce biased results, so the sliding speed is chosen as $v=1.0 \mathrm{~m} / \mathrm{s}$ here. The tip moves to right for $160 \Delta s$ to finish the sliding contact processes.

\subsection{Sliding Contacts of Model I}

For model I $(113 \times 81$ atoms $)$, Figure 3 shows the displacements in $y$ direction of atoms on the centerline of the substrate after the indentation. The COD is calculated according to Eq. (4), and $k=61$ corresponding

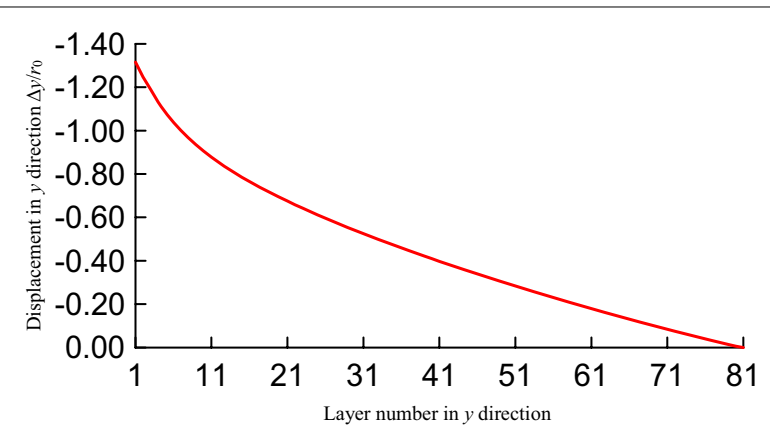

Figure 3 Displacements in y direction of atoms on the centerline of the substrate $(113 \times 81)$

to $C_{\mathrm{d}}=95.6 \%$. So, 61 layers in $y$ direction is selected, and the scale of model I for the sequent sliding contact is changed to $113 \times 61$ atoms. Friction forces from the LRMD simulation are given and compared with full MD simulation $(113 \times 81$ atoms $)$ in Figure 4 . The results from the two methods agree well with each other, which testifies the validity of the LRMD simulation method. Additionally, the period of fluctuation is $1.125 r_{0}(=45 \Delta s)$, and the distance between two adjacent atoms in $x$ direction is $1.122 r_{0}\left(=2^{1 / 6} r_{0}\right.$ for FCC Cu$)$. The difference between the two values is caused by the displacement increment.

\subsection{Sliding Contacts of Model II}

For model II $(227 \times 163$ atoms), Figure 5 shows the displacements in $y$ direction of atoms on the centerline of the substrate after the indentation. The COD is calculated according to Eq. (4), and $k=123$ corresponding to $C_{\mathrm{d}}=95.1 \%$. Then, the scale of the model for the sequent sliding contact is $227 \times 123$ atoms. During the whole sliding process, friction forces from the LRMD simulation and from full MD simulation are compared with each other in Figure 6. The agreement between two curves verifies the effectiveness of the LRMD simulation method further. Besides, the period of the fluctuation is also $1.125 r_{0}(=45 \Delta s)$, which is same as the case of Model I.

\subsection{Three-dimensional Model for Sliding Contacts}

To testify the idea further, a three-dimensional MD model is given in Figure 7. The model includes a rigid cylindrical tip and an elastic substrate (monocrystal copper with $\left[\begin{array}{lll}0 & 0 & 1\end{array}\right]$ surface). The tip consists of 3200 atoms with the radius $R=100 d$ ( $d$ is the minimum interatomic distance, $d=0.2556 \mathrm{~nm}$ ). The dimension of the substrate is $60 a_{0} \times 4 a_{0} \times 25 a_{0}\left(a_{0}\right.$ is the equilibrium lattice constant, for FCC Cu, $a_{0}=0.3615 \mathrm{~nm}$ ), corresponding to $x, y, z$ directions, and the total number of atoms of the substrate is 24000. Initial gap between the tip and substrate is $d_{\mathrm{g}}=2.2 d$. 


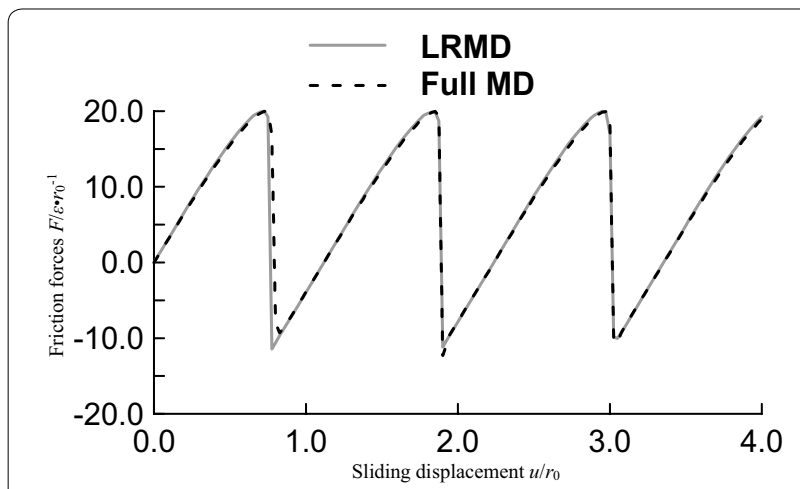

Figure 4 Friction forces comparison between LRMD simulation and full MD simulation $(113 \times 81)$

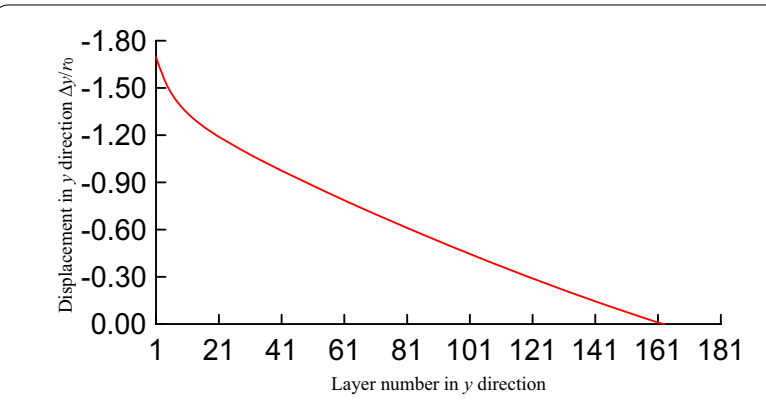

Figure 5 Displacements in $y$ direction of atoms on the centerline of the substrate $(227 \times 163)$

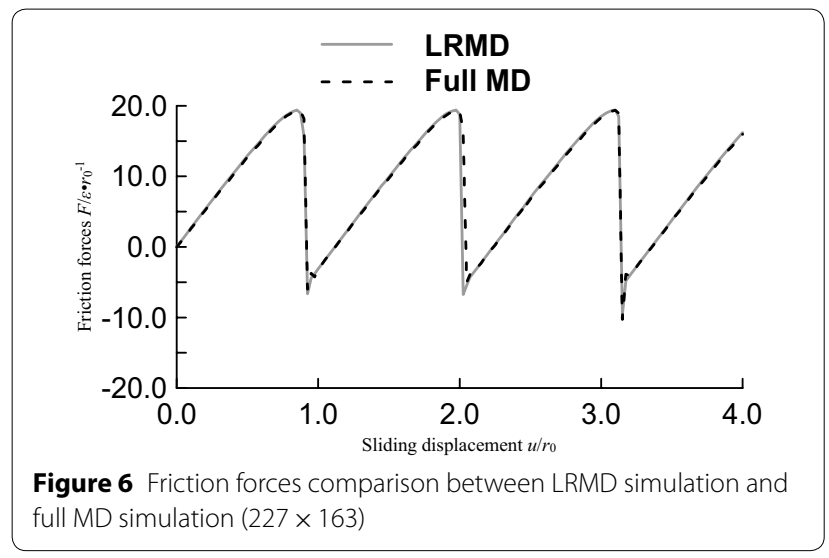

Fixed boundary conditions are applied on the bottom two layers, and periodic boundary conditions are applied along the $y$-axis and the two vertical boundaries of the $x O z$ section of the substrate. Embedded-atom method (EAM) is employed to describe the interactions between all atoms. Velocity-Verlet algorithm is carried out with a fixed time step, $\Delta t=5 \mathrm{fs}$. The temperature of the system is $T=300$ $\mathrm{K}$. The system will be relaxed for $2000 \Delta t$ to reach its minimum energy configuration before loading. Then, the tip moves to the substrate with a displacement increment $\Delta s=0.02 d$ and the indentation depth is $d_{\mathrm{I}}=0.4 d$. When the indentation is finished, the tip slides along $x$ direction, and the relaxation time is also $2000 \Delta t$. The tip moves to right for $200 \Delta s$ to finish the sliding contact processes.

After the indentation, the COD can be obtained by Eq. (4), and the dimension is $19 a_{0}$ in $z$ direction corresponding to $C_{\mathrm{d}}=95.7 \%$. So, the scale of the following model for sliding contact is $60 a_{0} \times 4 a_{0} \times 19 a_{0}$. Figure 8 compares the friction forces from full MD simulation $\left(60 a_{0} \times 4 a_{0} \times 25 a_{0}\right)$ and LRMD simulation $\left(60 a_{0} \times 4 a_{0} \times 19 a_{0}\right)$. For the full MD simulation, the maximum friction force is 5.302 , and the value is 4.805 for the LRMD simulation. The difference between the maximum friction forces is $9.37 \%$, which shows the effectiveness of the LRMD simulation method. Due to the indentation depth is small, friction forces are mainly influenced by adhesive forces. For full MD model, the deformation of the substrate will revert to some extent, while the reversion for the LRMD model will be lower than the full MD model due to fixed boundary conditions on the bottom two layers of the LRMD model. Lower reversion makes lower adhesive forces in the opposite direction of sliding, so the friction forces show the difference between LRMD and full MD. From Figure 8, one can also find that the periods of the fluctuation of these two curves are all $1.4 d(=70 \Delta s)$. This period approximates to the lattice constant $a_{0}$, and the difference between the two values should be also caused by the displacement increment. The phenomenon agrees well with the conclusion of Ref. [33], which shows validity of the LRMD simulation method.

\section{Discussion}

For two-dimensional sliding contact problems shown in Figure 9, friction force can be written as [34]:

$$
F=F_{\text {ploughing }}+F_{\text {adhesion }}
$$

where $F$ is total friction force. $F_{\text {ploughing }}$ and $F_{\text {adhesion }}$ are ploughing component and adhesion component, respectively. For a given load

$$
F_{\text {ploughing }} \infty \sqrt{\frac{1}{2(R / \delta)-1}}
$$

where $\delta$ is scratching depth, and $R$ is the radius of the cylindrical tip. For the same cylindrical tip, $F_{\text {ploughing }}$ increases as the increasing of $\delta$ according to Eq. (6). In this work, displacements in $y$ direction of the substrate atoms caused by the indentation will influence $\delta$ directly. For the models studied in this paper, the simulations consist of two main processes including indentation process and sliding process. The number of layers in indentation 


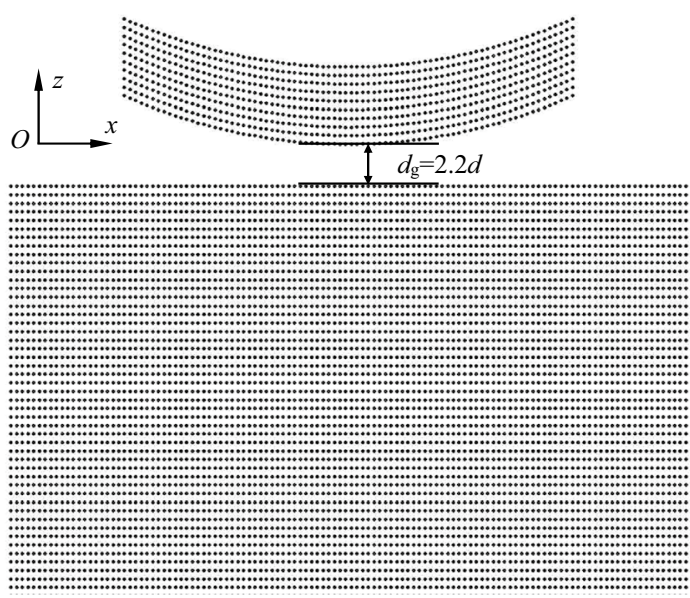

Figure 7 Three-dimensional nanoscale sliding contact model between a rigid cylindrical tip and an elastic substrate

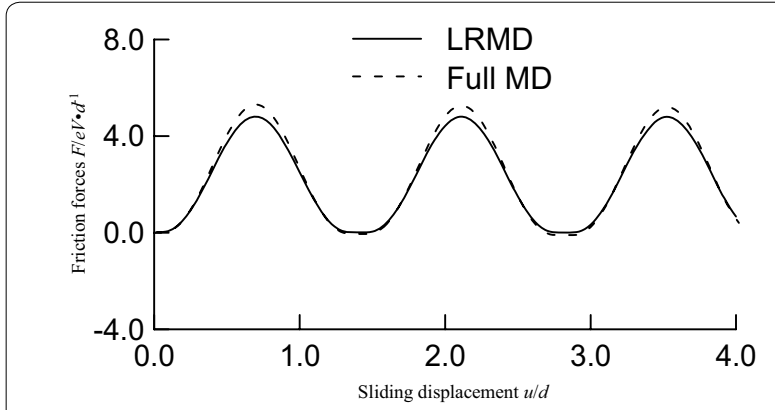

Figure 8 Friction forces comparison between LRMD simulation and full MD simulation $\left(60 a_{0} \times 4 a_{0} \times 25 a_{0}\right)$

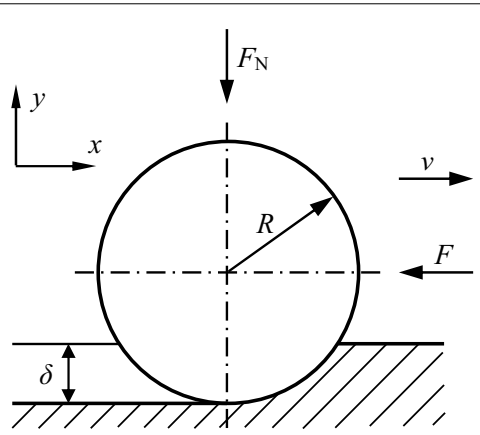

Figure 9 Schematic diagram for sliding contact problems

direction plays an important role in the indentation process. After indentation $\left(d=2.5 r_{0}\right.$, two-dimensional model for example), Figure 10 compares the displacements in $y$ direction of the first layer atoms for two different full
MD models: $113 \times 81$ and $113 \times 61$ atoms. The displacements in $y$ direction for the small model $(113 \times 61)$ are lower than the large model $(113 \times 81)$. From this figure, one can find that the displacements of each atom is different, and a dent occurs on surface of the original flat substrate. Contact areas are nearly the same for the two models, and adhesion component $F_{\text {adhesion }}$ is proportional to the contact area. So, one can infer that there is little difference between two models for adhesion components, and $F_{\text {ploughing }}$ will be the main role to induce the difference between total friction forces in the sliding processes under this indentation depth.

Comparing Figure 9 and Figure 10, the scratching depths of the two models are not as ideal as Figure 9. Equivalent scratching depth can be defined as follows:

$$
\delta_{\mathrm{e}}=\bar{y}-y_{\min }
$$

where $\bar{y}$ is average value of Y-coordinate of the first layer atoms after indentation, and $y_{\min }$ is the minimum value of $Y$-coordinate of the first layer atoms after indentation. They can be obtained as:

$$
\begin{aligned}
& \bar{y}=\frac{1}{n_{\text {layer }}} \sum_{i=1}^{n_{\text {layer }}}\left(y_{\mathrm{o}}-\left|\Delta y_{i}\right|\right) \\
& y_{\min }=y_{\mathrm{o}}-\left|\Delta y_{\max }\right|
\end{aligned}
$$

where $y_{0}$ is the original value of $Y$-coordinate of the first layer atoms, $\Delta y_{i}$ is the displacement in $y$ direction of atom $i$ on the first layer, $\Delta y_{\max }$ is the maximum displacement in $y$ direction of the first layer atoms, and $n_{\text {layer }}$ is total number of atoms on the first layer. Then, Eq. (7) can be rewritten as

$$
\delta_{\mathrm{e}}=\bar{y}-y_{\min }=\left|\Delta y_{\max }\right|-\frac{1}{n_{\text {layer }}} \sum_{i=1}^{n_{\text {layer }}}\left|\Delta y_{i}\right|
$$

By using Eq. (10) and the data from Figure 10, the equivalent scratching depths for the two models can be obtained, and the values are $0.767 r_{0}(113 \times 81)$ and $0.780 r_{0}(113 \times 61)$, respectively. The smaller model gets higher equivalent scratching depth, which will induce higher ploughing components and higher friction forces under the same initial conditions. Therefore, one should not use an original small model to simulate the large scale sliding contact problems due to different equivalent scratching depth, while LRMD simulation method can be used instead due to its same indentation model and same equivalent scratching depth. 


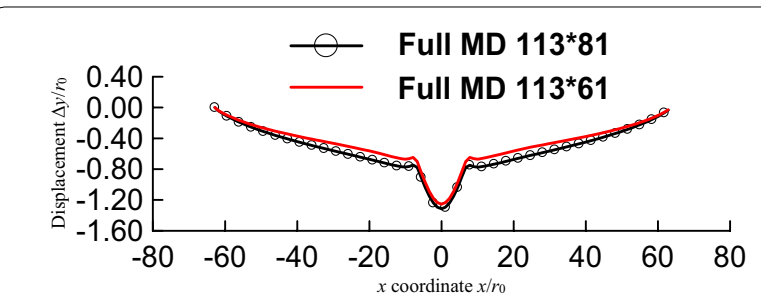

Figure 10 Comparison of displacements of the first layer atoms for different two-dimensional full MD models

\section{Conclusions}

Based on the contribution of displacement, a local region molecular dynamics simulation method is developed in this study. Two-dimensional and three-dimensional nanoscale sliding contacts between rigid cylindrical tips and elastic substrates with different scales are used as examples to testify the validity of the LRMD simulation method. Some conclusions are drawn as follows.

(1) The contribution of displacement can be a criterion used to estimate the effective local region during indentation process.

(2) LRMD simulation method can be used to solve nanoscale sliding contacts effectively, while original small MD model cannot be used to simulate the large scale sliding contact problems due to different equivalent scratching depth.

(3) For the same indentation depth, the scale of the model influences the equivalent scratching depth, and smaller model induces higher equivalent scratching depth as well as higher friction forces.

(4) Compared with full MD simulation, LRMD simulation method could save $35.67 \%$ CPU time.

\section{Authors' Contributions}

R-TT and GL were in charge of the whole trial; R-TT wrote the manuscript; T-XL assisted with sampling and laboratory analyses. All authors read and approved the final manuscript.

\section{Author Details \\ ${ }^{1}$ Shaanxi Engineering Laboratory for Transmissions and Controls, North- western Polytechnical University, Xi'an 710072, China. ${ }^{2}$ Institute for Mechan- ics and Fluid Dynamics, TU Bergakademie Freiberg, Lampadiusstr. 4, 09596 Freiberg, Germany.}

\section{Authors' Information}

Rui-Ting Tong, born in 1981, is currently an associate professor at Northwestern Polytechnical University, China. His research interests include space tribology, multiscale method, contact mechanics, nanoscale friction, etc

Geng Liu, born in 1961, is currently a professor at Northwestern Polytechnical University, China. His research interests include dynamics of mechanical system, tribology, contact mechanics, planetary roller screw mechanism, multiscale method, etc.

Tian-Xiang Liu, born in 1976, is currently a research fellow at Institute for Mechanics and Fluid Dynamics, Germany. His research interests include meshfree method, contact mechanics, nanoscale friction, etc.

\section{Competing Interests}

The authors declare that they have no competing interests.

\section{Funding}

Supported by National Natural Science Foundation of China (Grant Nos 51675429, 51205313), Fundamental Research Funds for the Central Universities of China (Grant No. 3102014JCS05009), and 111 Project of China (Grant No. B13044)

\section{Publisher's Note}

Springer Nature remains neutral with regard to jurisdictional claims in published maps and institutional affiliations.

Received: 15 May 2018 Accepted: 18 October 2018

Published online: 31 October 2018

\section{References}

[1] L Verlet. Computer "experiments" on classical fluids: I thermodynamical properties of Lennard-Jones molecules. Physical Review, 1967, 159(98): 98-103.

[2] RW Hockney, JW Eastwood. Computer simulations using particles. New York: McGraw-Hill, 1981.

[3] D Frenkel, B Smit. Understanding molecular dynamics-from algorithms to applications. San Diego: Academic Press, 1996.

[4] S B Kylasa, H M Aktulga, A Y Grama. Reactive molecular dynamics on massively parallel heterogeneous architectures. IEEE Transactions on Parallel and Distributed Systems, 2017, 28(1): 202-214.

[5] Y Hirokawa, N Nishikawa, T Asano, et al. A study of real-time and 100 billion agents simulation using the Boids model. Artificial Life and Robotics, 2016, 21(4): 525-530.

[6] Z Yao, J Wang, G Liu, et al. Improved neighbor list algorithm in molecular simulations using cell decomposition and data sorting method. Computer Physics Communications, 2004, 161 (1-2): 27-35.

[7] D R Mason. Faster neighbor list generation using a novel lattice vector representation. Computer Physics Communications, 2005, 170(1): 31-41.

[8] M Kim, K Choe, G Go, et al. A parallel algorithm for electrostatic interactions based on Wolf method charge-neutral condition and modified cell-linked list method. 2015 5th International Conference on Information Science and Technology (ICIST), Changsha, Hunan, China, April 24-26, 2015: 1-6.

[9] T Nagai, TTakahashi. Mass-scaling replica-exchange molecular dynamics optimizes computational resources with simpler algorithm. The Journal of Chemical Physics, 2014, 141(11): 114111(1-10).

[10] U Borštnik, B T Miller, B R Brooks, et al. Implementation of the force decomposition machine for molecular dynamics simulations. Journal of Molecular Graphics and Modelling, 2012, 38: 243-247.

[11] Y L Wu, X H Xu, X J Yang, et al. MDSLB: A new static load balancing method for parallel molecular dynamics simulations. Chinese Physics $B$, 2014, 23(2): 028903(1-16).

[12] C Begau, G Sutmann. Adaptive dynamic load-balancing with irregular domain decomposition for particle simulations. Computer Physics Communications, 2015, 190: 51-61.

[13] S Seckler, N Tchipev, H-J Bungartz, et al. Load balancing for molecular dynamics simulations on heterogeneous architectures. 2016 IEEE 23rd International Conference on High Performance Computing, Hyderabad, India, December 19-22, 2016: 101-110.

[14] D Davydov, J-P Pelteret, P Steinmann. Comparison of several staggered atomistic-to-continuum concurrent coupling strategies. Computer Methods in Applied Mechanics and Engineering, 2014, 277: 260-280.

[15] K Fackeldey. Challenges in atomistic-to-continuum coupling. Mathematical Problems in Engineering, 2015, 2015: 834517(1-10).

[16] T D Scheibe, E M Murphy, XY Chen, et al. An analysis platform for multiscale hydrogeologic modeling with emphasis on hybrid multiscale methods. Groundwater, 2015, 53(1): 38-56.

[17] E Biyikli, Q C Yang, A C To. Multiresolution molecular mechanics: dynamics. Computer Methods in Applied Mechanics and Engineering, 2014, 274 : $42-55$. 
[18] E Biyikli, A C To. Multiresolution molecular mechanics: adaptive analysis. Computer Methods in Applied Mechanics and Engineering, 2016, 305: 682-702.

[19] E Biyikli, A C To. Multiresolution molecular mechanics: implementation and efficiency. Journal of Computational Physics, 2017, 328: 27-45.

[20] YXWang, K-H Chang. Continuum-based shape sensitivity analysis for 2D coupled atomistic/continuum simulations using bridging scale decomposition. Mechanics Based Design of Structures and Machines, 2015, 43(2): 232-264.

[21] YXWang, K-H Chang. Continuum shape sensitivity analysis and what-if study for two-dimensional multi-scale crack propagation problems using bridging scale decomposition. Structural and Multidisciplinary Optimization, 2015, 51(1): 59-87.

[22] A Sadeghirad, F Liu. A three-layer-mesh bridging domain for coupled atomistic-continuum simulations at finite temperature: formulation and testing. Computer Methods in Applied Mechanics and Engineering, 2014, 268: 299-317

[23] A Tabarraei, X Wang, A Sadeghirad, et al. An enhanced bridging domain method for linking atomistic and continuum domains. Finite Elements in Analysis and Design, 2014, 92: 36-49.

[24] J Cho, T Junge, J-F Molinari, et al. Toward a 3D coupled atomistic and discrete dislocation dynamics simulation: dislocation core structures and Peierls stresses with several character angles in FCC aluminum. Advanced Modeling and Simulation in Engineering Sciences, 2015, 2(12): 1-17.

[25] E G Karpov, HYu, H S Park, et al. Multiscale boundary conditions in crystalline solids: theory and application to nanoindentation. International Journal of Solids and Structures, 2006, 43(21): 6359-6379.

[26] B Q Luan, S Hyun, J F Molinari, et al. Multiscale modeling of two-dimensional contacts. Physical Review E, 2006, 74(4): 046710(1-11).
[27] P R Budarapu, R Gracie, S P A Bordas, et al. An adaptive multiscale method for quasi-static crack growth. Computational Mechanics, 2014, 53(6): 1129-1148.

[28] RTTong, G Liu, TX Liu. Multiscale analysis on two dimensional nanoscale sliding contacts of textured surfaces. ASME Journal of Tribology, 2011, 133(4): 041401(1-13).

[29] K Maekawa, A Itoh. Friction and tool wear in nano-scale machining - a molecular dynamics approach. Wear, 1995, 188(1-2): 115-122.

[30] V Pandurangan, $\mathrm{T} \mathrm{Ng}, \mathrm{H}$ Li. Nanoscratch simulation on a copper thin film using a novel multiscale model. Journal of Nanomechanics and Micromechanics, 2013, 4(2): A4013008(1-8).

[31] P M Agrawal, B M Rice, D L Thompson. Predicting trends in rate parameters for self-diffusion on FCC metal surfaces. Surface Science, 2002, 515(1): 21-35.

[32] W C Swope, H C Andersen, P H Berens, et al. A computer simulation method for the calculation of equilibrium constants for the formation of physical clusters of molecules: application to small water clusters. The Journal of Chemical Physics, 1982, 76(1): 637-649.

[33] L C Zhang, K L Johnson, W C D Cheong. A molecular dynamics study of scale effects on the friction of single-asperity contacts. Tribology Letters, 2001, 10(1-2): 23-28.

[34] D F Moore. Principles and applications of tribology. Oxford: Pergamon Press, 1975.

\section{Submit your manuscript to a SpringerOpen ${ }^{\circ}$ journal and benefit from:}

- Convenient online submission

- Rigorous peer review

- Open access: articles freely available online

- High visibility within the field

- Retaining the copyright to your article

Submit your next manuscript at springeropen.com 